\title{
Desafíos de la producción multimedia en los MOOC. Estudio de caso interpretativo sobre las perspectivas docentes
}

\section{(Challenges of Multimedia Production in MOOCs. An Interpretive Case Study on the Faculty Perspectives)}

\author{
Ada Freitas-Cortina \\ Joaquín Paredes-Labra \\ Universidad Autónoma de Madrid, UAM (España)
}

DOI: https://doi.org/10.5944/ried.25.1.30840

\section{Cómo referenciar este artículo:}

Freitas-Cortina, A., y Paredes-Labra, J. (2022). Desafíos de la producción multimedia en los MOOC. Estudio de caso interpretativo sobre las perspectivas docentes. RIED. Revista Iberoamericana de Educación a Distancia, 25(1), pp. 59-79. https://doi.org/10.5944/ried.25.1.30840

\section{Resumen}

En un contexto enmarcado por un proceso imparable de digitalización de enseñanza superior, los entornos de aprendizaje abiertos y masivos, del acrónimo MOOC (Massive Open Online Courses), se han convertido en un catalizador de soluciones para mejorar el desarrollo profesional docente y las experiencias de aprendizaje de estudiantes. Los contenidos audiovisuales y multimedia siguen ocupando gran parte de la preocupación y relevancia en el diseño de estos cursos. El objetivo de este trabajo es entender cómo se da el empleo de los materiales y los recursos digitales utilizados en estos entornos digitales como medio para reflexionar sobre los desafíos actuales de la formación del profesorado en competencia audiovisual y mediática. A partir de métodos mixtos de investigación, se desarrolló un estudio de caso con enfoque interpretativo desde las experiencias de innovación de la Universidad Autónoma de Madrid (UAM). Se utilizaron análisis estadísticos de datos de la plataforma tecnológica, así como análisis del discurso pedagógico de las entrevistas al profesorado implicado en los proyectos de innovación. Con todo ello se realizó un proceso de triangulación e integración de los datos cuantitativos y cualitativos. Entre los principales hallazgos, se pone de manifiesto que la producción de píldoras de contenido audiovisual fue el aspecto más novedoso y a la vez más desafiante del desarrollo de proyectos MOOC. Resulta fundamental más inversión institucional para trabajar las dificultades que implican la alfabetización audiovisual y mediática del profesorado.

Palabras clave: MOOC; medios audiovisuales; medios de enseñanza; desarrollo profesional. 


\begin{abstract}
In a context framed by an unstoppable process of digitization of higher education, the Massive Open Online Courses (MOOC) has become a catalyst for solutions to improve teacher professional development and student learning experiences. The audiovisual and multimedia contents continue to occupy a large part of the concern and relevance in the design of these courses. The objective of this work is to understand how the use of digital materials and resources applied in these digital environments occurs as a means of reflecting on the current challenges of teacher training in audiovisual and media competence. Using mixed research methods, an interpretive case study was developed from the innovation experiences of the Autonomous University of Madrid (UAM). Statistical data analysis of the technological platform was used, as well as analysis of the pedagogical discourse of the interviews with the faculty involved in the innovation projects. With all this, was carried out a process of triangulation and integration of quantitative and qualitative data. Among the main findings, it is shown that the production of audiovisual content pills was the most innovative and at the same time the most challenging aspect of the development of MOOC projects. More institutional investment is essential to address the difficulties that the audiovisual and media literacy of teachers imply.
\end{abstract}

Keywords: MOOC; audiovisual aid; teaching aid; career development.

El contexto universitario actual está enmarcado por un proceso imparable de transformación digital de la enseñanza superior (Adell et al., 2018; Chinkes y Julien, 2019), en consecuencia a la evolución del desarrollo tecnológico de los entornos e-learning y sus nuevas herramientas de comunicación y colaboración en línea (Bai et al., 2021; Downes, 2012).

En respuesta a la imposición de la digitalización de las instituciones de educación superior (Demetriadis et al., 2018; Hollands y Tirthali, 2014), el fenómeno de los entornos de aprendizaje abiertos y masivos, conocido por el acrónimo MOOC (Massive Open Online Courses) u otras variaciones, ha impactado de manera definitiva a los escenarios de educación superior. La problematización de sus principales implicaciones lo ha convertido en un catalizador de soluciones de la educación abierta y flexible (Daniel y Uvalić-Trumbić, 2014; Sancho et al., 2018) para promover la mejora del desarrollo profesional docente (Czerniewicz et al., 2016; Mallmann y Nobre, 2017; Misra, 2018) y las experiencias de aprendizaje de los estudiantes (Anderson y Dron, 2012; Zhao et al., 2020).

Los MOOC se caracterizan en un entorno de aprendizaje predominantemente basado en la escalabilidad y la accesibilidad (Moe, 2015), que se alimenta de las interacciones de miles de estudiantes y que permite la autogestión del propio aprendizaje. En tales entornos, el aprendizaje se caracteriza por la interacción con micro-estructuras (micro-contenidos, micro-formatos, micro-lecturas), lo que se denomina el fenómeno del "micro-aprendizaje" (Silva y Souza, 2016). 
Los materiales y los contenidos son las principales preocupaciones de las investigaciones que se han desarrollado sobre el diseño de los MOOC (Cabero y Llorente, 2017). En sus diseños, se encuentran con frecuencia las videoconferencias, los cuestionarios, las tareas y ejercicios, los foros de discusión y un examen final (Lin, 2017). No obstante, el principal medio de enseñanza sigue siendo las conferencias, grabadas en formato audiovisual, pero más ágiles y cortas, que duran de 8 a 12 minutos y son impartidas por los profesores de renombre (Gértrudix Barrio et al., 2017; Mallmann y Nobre, 2017).

Los vídeos son ampliamente reconocidos como los principales materiales en los MOOC y su calidad suele influir en gran medida en el nivel de participación de los estudiantes (Guo et al., 2014; Lemay y Doleck, 2020; Lin, 2017). De ahí, los profesores MOOC suelen usar la estrategia de fragmentar el tema de las videoconferencias con preguntas intercaladas para verificar si el espectador ha captado el contenido anterior o puede aplicarlo a un problema (Hollands y Tirthali, 2014). Además, en la grabación de los vídeos es fundamental que el profesor posea un buen dominio de diferentes técnicas de comunicación y presentación de la información y habilidades relacionadas con las antiguas artes de la oratoria y la retórica (Corny, 2021; Mallmann y Nobre, 2017).

Asimismo, son muchas las propuestas innovadoras que pueden darse en estos entornos digitales. Por ello, Conole (2016) propone clasificarlos según un conjunto de doce dimensiones que refleje mejor la complejidad de las innumerables posibilidades de diseño de un MOOC. No obstante, algunos autores (Bartolomé y Steffens, 2015; Drake et al., 2015; Guàrdia et al., 2013) están de acuerdo con lo que señala Lane (2012): un MOOC generalmente manifiesta tendencias que ponen acento en la conexión en red (cMOOC), en las tareas (tMOOC) o en el contenido (xMOOC) en función de las bases teórico-pedagógicas que fundamentan su diseño (conectivista, constructivista y cognitivista, respectivamente).

Por otra parte, uno de sus impactos más relevantes se trata del potente cambio en las relaciones entre el profesor, el contenido y el estudiante (Siemens, 2013): esa interacción tradicional se incrementa cada vez más con la utilización de los recursos educativos abiertos (REA) y su incentivo a la co-creación colaborativa entre los estudiantes periféricos y los expertos externos al proceso del aprendizaje en línea.

Los autores Anderson y Mcgreal (2012) señalan dos estrategias para darse este cambio de interacciones entre profesor-estudiante: (1) usar la facilidad de los medios digitales para producir los propios blogs, vídeos y podcasts en los canales y servicios de WordPress, Youtube, Khan Academy, etc., convirtiendo las interacciones de las lecciones magistrales, discusiones, conferencias o experimentos en contenidos educativos iterativos; (2) aumentar la calidad y la frecuencia de la interacción estudiante-estudiante a través de las tecnologías digitales y sus servicios de intercambio, comunicación y colaboración en red.

No obstante, los estudios recientes sobre los MOOC (Giasiranis y Sofos, 2020; Zhao et al., 2020; Zhu et al., 2018) apuntan que en lugar de ofrecer propuestas 
de actividades que impliquen la participación de los estudiantes en procesos de colaboración, reutilización, remezcla, redistribución, inclusión y adaptación de contenidos, la mayoría de sus diseños se orientan hacia las plataformas tecnológicas con una estructura predeterminada, secuencial e individual. Eso pone en evidencia que sus contenidos no contemplan los principios de apertura y las nuevas pedagogías que estos entornos suponen (Chiappe-Laverde et al., 2015; Sánchez-Vera et al., 2015; Zapata-Ros, 2013).

Por lo tanto, existe una gran dificultad de transformar el discurso pedagógico sobre la educación abierta en buenas prácticas educativas para la creación de espacios más interesantes que cambien la manera en la que los estudiantes y los profesores interactúan y se relacionan a través de los contenidos audiovisuales e interactivos (Ramírez-Fernández et al., 2015).

Por ello, muchos estudios (Callejo-Gallego y Agudo-Arroyo, 2018; Gehrke y Kezar, 2015; Gértrudix Barrio et al., 2017; Gil-Jaurena y Domínguez, 2018; Mallmann y Nobre, 2017) señalan la necesidad de que las instituciones de educación superior ofrezcan el apoyo y las estructuras necesarias al desarrollo de los nuevos roles y responsabilidades que se han incorporado a la labor del profesorado, en particular en los entornos virtuales y producción audiovisual, en el marco de la reconstrucción de su identidad y docencia digital.

Para hacer frente a ese gran desafío, hacen falta más investigaciones sobre el diseño pedagógico y las experiencias de aprendizaje que promueven los contenidos audiovisuales e interactivos de los MOOC desde la perspectiva del profesorado como principal agente del cambio pedagógico (Armellini y Padilla-Rodriguez, 2016; Sangrà et al., 2015; Toven-Lindsey et al., 2015). Un estudio con tal enfoque requiere un análisis de las transformaciones que emergen de las experiencias innovadoras apoyadas en estos nuevos entornos de aprendizaje, indagando si las experiencias docentes a través de los MOOC o SPOC modifican la forma de diseñar sus materiales y recursos y si mejoran las prácticas docentes.

No obstante, investigar en profundidad las implicaciones relacionadas con las prácticas abiertas no es un proceso sencillo (Chiappe-Laverde et al., 2015; Siemens, 2013; Zapata-Ros, 2013) debido a la complejidad misma que implican los procesos de innovación (Ramírez-Montoya y Lugo-Ocando, 2020; Sancho, 2018) y la dificultad de determinar las prácticas docentes que favorezcan el aprendizaje en línea como proceso de actuación y diálogo colectivo (García Peñalvo y Corell, 2020; Kuzu, 2020; Salinas et al., 2008).

Asimismo, a medida que más profesores universitarios comiencen a enfrentarse a los desafíos de los entornos de aprendizaje en línea abiertos y masivos, se espera que su reflexión se centre más en las preocupaciones sobre el acceso al contenido audiovisual e interactivo, los modelos pedagógicos efectivos, las posibilidades del aprendizaje abierto en línea y el potencial de los recursos educativos abiertos (Czerniewicz et al., 2016; Nascimbeni y Burgos, 2016; Siemens, 2013) para que la comunidad académica pueda apreciar mejor las posibilidades de la educación en 
línea más abierta y flexible, dando forma a sus futuras direcciones (Ebben y Murphy, 2014; Ramírez-Montoya, 2020; Vázquez-Cano et al., 2021).

\section{OBJETIVOS}

El objetivo principal fue entender los cambios producidos en los materiales y los recursos utilizados en las experiencias docentes por parte del profesorado implicado en prácticas innovadoras en los nuevos entornos digitales de aprendizaje en red, así como la influencia de la infraestructura y disponibilidad a los servicios de apoyo ofrecidos por la universidad.

Por ello, se establecen los principales interrogantes de la investigación: ¿Qué estrategias metodológicas, materiales curriculares, interacciones didácticas y enfoques de evaluación pone en marcha el profesorado en los MOOC? ¿Cómo cambia el diseño de contenidos y el material/recurso empleado? ¿Cómo se caracteriza el servicio de apoyo de la unidad central y el apoyo del equipo técnico? ¿Cuál es el peso de las plataformas en condicionar los diseños implementados?

A partir de estos interrogantes, se desarrolló un estudio de caso con enfoque interpretativo de estrategia mixta para profundizar acerca de los cambios en los procesos pedagógicos relacionados con la producción de contenidos audiovisuales y multimedia en entornos digitales a través de las experiencias innovadoras del profesorado en los proyectos MOOC ofertados por la Unidad de Tecnologías para la Educación (UTED) de la Universidad Autónoma de Madrid (UAM).

El estudio forma parte de una investigación más amplia sobre cómo estos programas de innovación docente han influido en el comportamiento, práctica y concepciones de profesores y estudiantes, así como en la estrategia organizacional, para la mejora de los procesos de enseñanza superior y su transformación digital.

A través del caso de la UAM, se ha pretendido brindar una línea de reflexión sobre la problematización de la digitalización de las universidades desde el punto de vista del profesorado universitario, desde un enfoque de la sociología digital de Neil Selwyn (2018) que pudiera servir de referencia a otros contextos universitarios con similares características y que se enfrentan al mismo desafío.

\section{METODOLOGÍA}

El estudio de caso (Stake, 2010) con enfoque metodológico interpretativo (Flick, 2002) se basó en el conocimiento práctico y personal (Elbaz, 1983; Tardif, 2004) del profesorado implicado en el diseño, creación y entrega de un MOOC en la plataforma edX del programa innovación tecnológica en la UAM.

Mediante la estrategia mixta de concurrencia paralela de recogida y análisis de datos cualitativos y cuantitativos (Creswel y Clark, 2017; Hernández-Sampieri y Mendoza, 2018), los procedimientos y técnicas empleados en el estudio se llevaron 
a cabo por medio de tres fases consecutivas (Figura 1): I. Acceso y preparación; II. Recogida y trabajo de campo; III. Análisis e interpretación.

\section{Figura 1}

Procedimientos de la estrategia mixta desarrollada en el estudio de caso

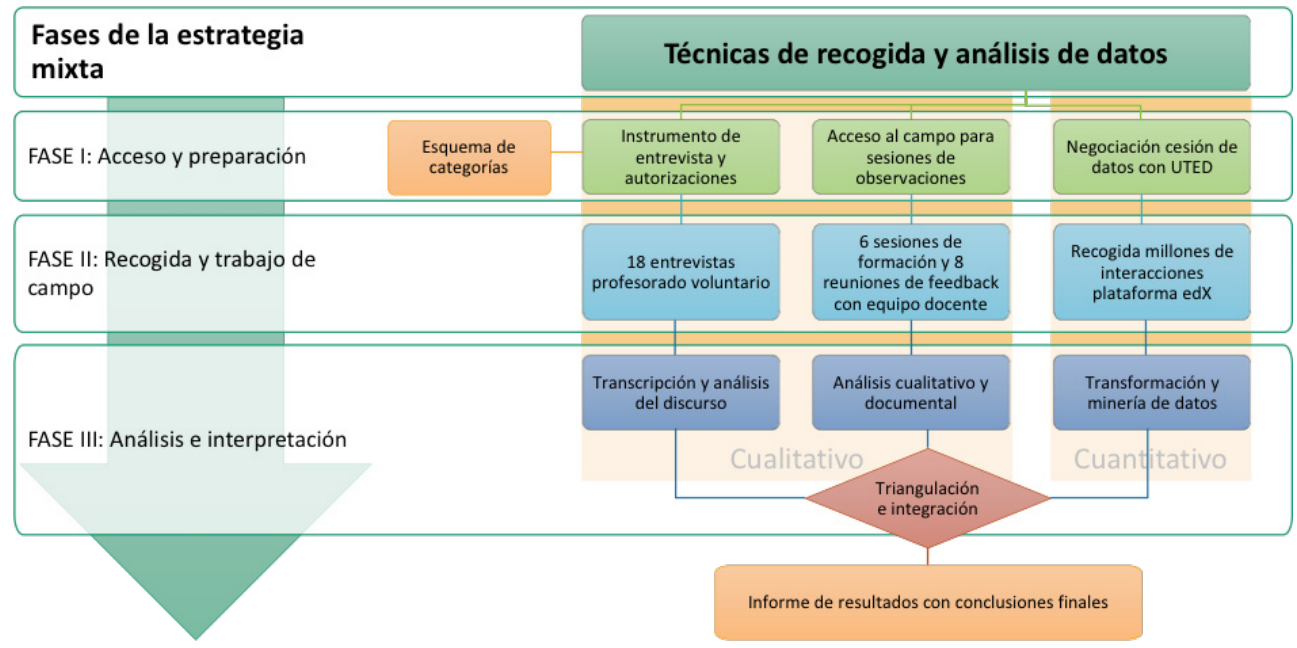

Durante el periodo del trabajo de campo, llevado a cabo en cuatro cursos académicos (2014-2015, 2015-2016, 2016-2017, 2017-2018), se han impartido entre 2 a 4 ediciones de los primeros 8 MOOC ofertados de forma escalonada por la universidad. No obstante, en 2017 es cuando se recogen los datos de la plataforma edX y se completan las entrevistas al profesorado participante en relación con todo el proceso vivenciado en este período.

En el método cuantitativo, se emplearon dos técnicas de recogida: los datos de los componentes y recursos de los cursos configurados en la plataforma edX.

Se accedió a los datos brutos de las dos primeras ediciones de los $8 \mathrm{MOOC}$ de la UAM, impartidos entre el período entre 2015 y 2016 (Tabla 1): Android301x, Equidad801x, Idealismo501x, Quijote501x, QuiOrg101x, Renal701x, SegAlim201x, TxEtj201x. Son estas primeras ediciones las que contienen las diferencias significativas en el diseño del curso (proceso iniciado en 2014 para algunos cursos), las siguientes ediciones se publican sin cambios en los contenidos. Estos cursos de diferentes temáticas implicaron la representación de todas las áreas de conocimiento: Artes y Humanidades (AH), Ciencias Sociales (CS), Ciencias Naturales (CN), Ciencias de la Salud (CSd), Ingeniería y Tecnologías (IT).

Se dispuso 8 ficheros en formato .rar, uno por cada proyecto MOOC, con los siguientes archivos de los datos anonimizados extraídos de la plataforma edX: 
COURSE con información general de los cursos y su estructura en ficheros .json y .sql; CSV con información del perfil de los usuarios y las interacciones en los foros; EVENTOS con logs diarios del tráfico de las interacciones de los usuarios anonimizados en formato .txt y .xml. A estos datos cuantitativos, se aplicó un proceso de reducción con una aplicación informática ad hoc desarrollada por el equipo de la universidad para el filtrado e integración de los datos requeridos de las dos ediciones de cada curso y un posterior volcado de toda la información en un único fichero CSV para el análisis estadístico descriptivo de las frecuencias absolutas y relativas en el programa IBM SPSS Statistics for Windows, versión 25.0 y su posterior representación gráfica en Excel para presentar la estructuración de los cursos, el perfil de los participantes, las inscripciones y progresos, así como las interacciones sociales en los foros y con el contenido de los cursos.

\section{Tabla 1}

Características de los MOOC implicados en el estudio del caso

\begin{tabular}{|c|c|c|c|c|c|}
\hline Título del MOOC & $\begin{array}{l}\text { Áreas del } \\
\text { conocimiento }\end{array}$ & Duración & Ediciones & Profesores & TA \\
\hline La España de El Quijote & $\begin{array}{l}\text { Artes y } \\
\text { Humanidades } \\
\text { (AH) }\end{array}$ & 7 semanas & 3 & 2 & 1 \\
\hline $\begin{array}{l}\text { Trasplante de órganos } \\
\text { - Desafíos éticos y } \\
\text { jurídicos }\end{array}$ & $\begin{array}{l}\text { Ciencias Sociales } \\
\text { (CS) / Ciencias } \\
\text { de la Salud (CSd) }\end{array}$ & 9 semanas & 3 & 2 & 1 \\
\hline $\begin{array}{l}\text { La Química Orgánica, un } \\
\text { mundo a tu alcance }\end{array}$ & $\begin{array}{l}\text { Ciencias } \\
\text { Naturales (CN) }\end{array}$ & 6 semanas & 3 & 1 & o \\
\hline $\begin{array}{l}\text { Jugando con Android - } \\
\text { Aprende a Programar tu } \\
\text { Primera App }\end{array}$ & $\begin{array}{l}\text { Ingenierías y } \\
\text { Tecnologías (IT) }\end{array}$ & 7 semanas & 4 & 5 & 2 \\
\hline $\begin{array}{l}\text { Fisiopatología renal } \\
\text { y enigmas de la vida } \\
\text { cotidiana }\end{array}$ & $\begin{array}{l}\text { Ciencias de la } \\
\text { Salud (CSd) }\end{array}$ & 6 semanas & 2 & 3 & 1 \\
\hline $\begin{array}{l}\text { Idealismo Filosófico: } \\
\text { Cómo hacer mundos con } \\
\text { ideas }\end{array}$ & $\begin{array}{l}\text { Artes y } \\
\text { Humanidades } \\
\text { (AH) }\end{array}$ & 5 semanas & 2 & 6 & 1 \\
\hline $\begin{array}{l}\text { Educación de calidad } \\
\text { para todos. Equidad, } \\
\text { inclusión y atención a la } \\
\text { diversidad. }\end{array}$ & $\begin{array}{l}\text { Ciencias Sociales } \\
\text { (CS) }\end{array}$ & 6 semanas & 2 & 4 & 2 \\
\hline $\begin{array}{l}\text { De la granja a la mesa: } \\
\text { La seguridad alimentaria } \\
\text { en la Unión Europea }\end{array}$ & $\begin{array}{l}\text { Ciencias Sociales } \\
\text { (CS) / Ciencias } \\
\text { Naturales (CN) }\end{array}$ & 6 semanas & 2 & 7 & 1 \\
\hline
\end{tabular}

Nota: el acrónimo TA se refiere a la figura del Teacher Assistant. 
En el método cualitativo, se emplearon tres técnicas de recogida: las entrevistas al profesorado, la observación de campo y el análisis documental.

Se realizaron entrevistas presenciales, individuales y semiestructuradas (Kvale, 2011) a 18 participantes voluntarios de un grupo de 30 profesores implicados en las dos ediciones de los $8 \mathrm{MOOC}$ del caso, que incluyeron al menos un representante de cada área del conocimiento. Se trató de un grupo diverso en género, tiempo de experiencia profesional y manejo de tecnologías, con escasa formación inicial en aspectos pedagógicos y con alguna formación previa sobre el MOOC recibida por el propio programa de innovación dela universidad(Tabla2). Asimismo, cabemencionar que el estudio incluye la perspectiva de profesores proclives a la innovación docente, con trayectoria en proyectos innovadores y experiencia investigación colegiada.

Para las entrevistas, se elaboró un instrumento validado por diez expertos en metodología cualitativa, formado por 7 hombres y 3 mujeres, vinculados al departamento de pedagogía de la UAM, que consistió en 32 preguntas basadas en las 4 categorías previas definidas, siendo 8 preguntas específicas sobre los aspectos de desarrollo de los materiales y recursos del proyecto MOOC puestos en marcha para establecer la interacción didáctica de los estudiantes.

\section{Tabla 2}

Características de los profesores participantes del estudio de caso

\begin{tabular}{|c|c|c|c|c|c|}
\hline $\begin{array}{c}\text { Área de } \\
\text { conocimiento }\end{array}$ & $\begin{array}{l}\text { Profesores } \\
\text { implicados }\end{array}$ & $\begin{array}{l}\text { Experiencia } \\
\text { docente }\end{array}$ & $\begin{array}{l}\text { Formación } \\
\text { pedagógica } \\
\text { inicial }\end{array}$ & $\begin{array}{l}\text { Conocimiento } \\
\text { de las TIC }\end{array}$ & $\begin{array}{l}\text { Formación } \\
\text { MOOC } \\
\text { recibida }\end{array}$ \\
\hline \multirow{4}{*}{$\begin{array}{l}\text { Artes y } \\
\text { Humanidades } \\
\text { (AH) }\end{array}$} & Profesora 2 & Novato & No & Intermedio & Sí \\
\hline & Profesor 7 & Novato & No & Avanzado & Sí \\
\hline & Profesor 8 & Maduro & No & Intermedio & Sí \\
\hline & Profesor 12 & Experto & No & Básico & No \\
\hline \multirow{6}{*}{$\begin{array}{l}\text { Ciencias } \\
\text { Sociales (CS) }\end{array}$} & Profesora 1 & Maduro & Sí & Básico & Sí \\
\hline & Profesora 3 & Maduro & No & Intermedio & No \\
\hline & Profesor 11 & Experto & Sí & Avanzado & Sí \\
\hline & Profesora 14 & Experto & Sí & Básico & Sí \\
\hline & Profesora 18 & Experto & Sí & Básico & Sí \\
\hline & Profesor 19 & Experto & Sí & Intermedio & Sí \\
\hline \multirow{2}{*}{$\begin{array}{l}\text { Ciencias } \\
\text { Naturales (CN) }\end{array}$} & Profesor 6 & Experto & No & Avanzado & No \\
\hline & Profesor 16 & Maduro & No & Básico & Sí \\
\hline
\end{tabular}




\begin{tabular}{llllll}
$\begin{array}{l}\text { Área de } \\
\text { conocimiento }\end{array}$ & $\begin{array}{c}\text { Profesores } \\
\text { implicados }\end{array}$ & $\begin{array}{c}\text { Experiencia } \\
\text { docente }\end{array}$ & $\begin{array}{l}\text { Formación } \\
\text { pedagógica } \\
\text { inicial }\end{array}$ & $\begin{array}{c}\text { Conocimiento } \\
\text { de las TIC }\end{array}$ & $\begin{array}{c}\text { Formación } \\
\text { MOOC } \\
\text { recibida }\end{array}$ \\
$\begin{array}{l}\text { Ciencias de la } \\
\text { Salud (CSd) }\end{array}$ & Profesora 5 & Novato & No & Intermedio & Sí \\
\cline { 2 - 6 } & Profesor 9 & Experto & No & Básico & Sí \\
\cline { 2 - 6 } $\begin{array}{l}\text { Ingenierías y } \\
\text { Tecnologías (IT) }\end{array}$ & Profesora 13 & Novato & No & Avanzado & Sí \\
\cline { 2 - 6 } & Profesora 4 & Maduro & No & Avanzado & Sí \\
\cline { 2 - 6 } & Profesor 17 & Maduro & No & Avanzado & No \\
\hline
\end{tabular}

Además, se realizó la observación de campo (Angrosino, 2012) a 14 sesiones de trabajo con los equipos docentes durante el desarrollo de los MOOC/SPOC y el análisis de la documentación relacionada con estos proyectos de innovación. La observación participante se dio en las 6 sesiones de formación docente durante la fase de diseño pedagógico de los MOOC y también en las 8 reuniones de evaluación, cierre y feedback de la primera edición de los MOOC ofertados por la oficina UAMx a lo largo del periodo del estudio de caso con cada equipo docente implicado para valorarse las leccione aprendidas en el proyecto MOOC de cara a la segunda edición del curso. Las sesiones tuvieron una duración media de 2 horas y se realizaron apuntes personales, interpretando la realidad observada. Las evidencias recogidas en las observaciones han ayudado a triangular y justificar los datos obtenidos de las entrevistas.

El análisis de los datos cualitativos y la documentación recogida se llevó a cabo en Atlas.ti versión 8.4.5 mediante procesos de codificación abierta, axial y selectiva (Strauss y Corbin, 2002), resultando en un total de 37 códigos y 453 citas codificadas a partir de las 4 categorías y sus 15 subcategorías previas definidas con relación a los aspectos más tangibles de los diseños de los recursos y materiales, los entornos y los servicios utilizados en la planificación de los proyectos MOOC de la UAM.

Por último, se realizó el proceso de triangulación e integración de los análisis cualitativos del discurso del profesorado como la principal fuente de información del estudio con los análisis complementarios cuantitativos de las características de los cursos para contrastar e interpretar los principales hallazgos del estudio (Hernández-Sampieri y Mendoza, 2018) sobre las estrategias de producción de los contenidos audiovisuales y multimedia en los entornos abiertos y masivos, así como sus aspectos favorecedores y limitadores de cara a la transferencia de estas a la docencia universitaria habitual. 


\section{RESULTADOS}

En los resultados, el análisis cualitativo y el proceso de codificación de la narrativa del discurso pedagógico del profesorado se presenta junto a las estadísticas descriptivas sobre el uso de los componentes pedagógicos disponibles en el diseño de los MOOC.

Mediante las representaciones de las relaciones entre las categorías de análisis y datos estadísticos, se extrajeron los principales hallazgos y conclusiones según los asertos más relevantes de diversidad de experiencias y perspectivas del profesorado participante, poniendo en evidencia las concepciones y contradicciones pedagógicas que emergen en el desarrollo del proyecto de innovación docente. A continuación, se presentan estos hallazgos en forma de los asertos más destacables (A-H) obtenidos de este proceso de triangulación de sus cuatro categorías.

\section{Aspectos característicos de los proyectos MOOC}

A. El aspecto más novedoso de los proyectos MOOC fue la incorporación de la enseñanza en línea escalable y dinámica a través de la adaptación del contenido a un formato más accesible e interactivo.

En primer lugar, los profesores destacaron algunas características específicas que consideraron como los aspectos más novedosos de los proyectos MOOC desarrollados, todos ellos relacionados a la aplicación de una metodología de enseñanza en línea escalable y dinámica mediante la incorporación de las tecnologías educativas, principalmente a través de los recursos multimedia y audiovisuales.

Pero lo que ha sido nuevo completamente han sido los vídeos, es decir, en los cursos que yo había participado anteriormente no había vídeos. [...] incluir un clip de vídeo entre demás contenidos textuales que sale como consecuencia de lo que se ha dicho en el vídeo, pero no exactamente lo mismo. Y eso me parece muy interesante, como mínimo decir en todo caso que sí es lo mismo, pero decirlo de otra manera completamente diferente. Ese es exactamente el objetivo. Y es algo que es completamente nuevo en esto de los MOOC y que a mí la verdad es que me ha encantado. (Profesor 6 de $\mathrm{CN}$ )

B. La oferta de los MOOC se caracterizó en cursos introductorios de diferentes temáticas con un carácter indagatorio y un sentido práctico al conocimiento transmitido.

Se observa que en todos los enunciados de los proyectos MOOC de la UAM (Tabla 1), además de un carácter indagatorio, hay un sentido práctico al conocimiento 
transmitido y un enfoque introductorio a las diferentes temáticas abordadas. Según la perspectiva del propio profesorado, ese enfoque práctico de la enseñanza requería centrarse en conceptos muy concretos e ir al grano en las explicaciones, para obtener un contenido simple, pero a la vez atractivo a todos los públicos, con opciones para la profundización en el tema: "[...] la respuesta que obtuvimos yo creo que acredita que era un tema actual, interesante y que para los alumnos resultó igualmente un temario que les creaba retos." (Profesor 11 de CS)

[...] sobre todo porque queríamos después mostrar cómo se estaba concretando en la práctica como medio para inspirar a otros para reflexionar e iniciar su propio proceso de transformación, de tal manera que si algo teníamos claro es que no queríamos hacer un curso teórico, sino dejar muy claro los conceptos de la base. (Profesora 14 de CS)

\section{Aspectos de la planificación y estructura de los cursos}

C. Al profesorado sin experiencia en la enseñanza en línea ni en la aplicación de metodologías activas a través de las tecnologías digitales, la planificación de la estructura del curso le supuso un gran desafío.

Con respeto a la fase de planificación y diseño de la estructura del curso, por lo general se observó que su desarrollo no fue un proceso lineal, dado que involucraba a un equipo docente sin experiencia en la plataforma tecnológica ni en las metodologías activas que estos entornos virtuales suponen, lo que ha demandado mucho apoyo del equipo técnico de la oficina UAMx en esta fase de toma de contacto.

Entre mi idea de algo que desconocía, yo creía que el MOOC era un poco como una plataforma Moodle donde colocas los materiales, y el equipo técnico fue el que me la hundió por completo, me dijo que habría que estructurarlo fundamentalmente de pie-arriba, estructurarlo de otra manera, vimos como funcionaba realmente el soporte a la plataforma edX y como tuve que recolocar los materiales de forma diferente al que yo lo pensaba. Hasta que me familiaricé con ella, pero el objetivo se ha mantenido [...]. (Profesor 12 de AH)

D. La estructuración del contenido MOOC implicó un proceso de revisión y síntesis del contenido más relevante, de carácter práctico y adaptado, a un formato digital corto, dinámico y a la vez interactivo.

Muchos de los profesores implicados en el proyecto MOOC comentaron que lo más difícil del proceso de planificación fue la estructuración del contenido, la 
construcción de una síntesis del contenido relevante, enfocado en ejemplos prácticos y adaptado a un formato digital corto, dinámico y a la vez interactivo.

[...] el problema era cómo adaptar el contenido al formato, entonces con la estructura en Excel que realizamos, que además era igual todas las semanas, implicaba una distribución de tiempos y una distribución de contenidos por personas y por temas, eso fue lo que nos salvó la vida. Eso nos costó, pero cuando conseguimos hacer la estructura fue uno de los mayores logros y lo que más ayudó más adelante. (Profesor $7 \mathrm{de} \mathrm{AH}$ )

Se hizo hincapié en el gran esfuerzo que supone la producción de las pequeñas píldoras de conocimiento práctico adaptadas a la dinámica y variada estructura del contenido del curso.

Hemos realizado sesiones internas de brainstorming entre el equipo docente y algunos estudiantes invitados para el diseño y el ajuste del contenido al nivel del público general. Después de definir la estructura, se desarrolló el material con mucha síntesis, a través de pequeñas píldoras de contenidos, para preparar un contenido definitivo y de calidad, seleccionando lo más importante del "qué decir”, eso requiere un esfuerzo previo para elaboración de los textos de los guiones de los vídeos y materiales de apoyo. (Profesora 13 de CSd)

\section{Aspectos de la producción de los materiales y recursos}

E. El contenido audiovisual y multimedia fue el principal material utilizado en los cursos.

Basándose en los análisis estadísticos de los datos recogidos desde la plataforma edX (Figura 2), a pesar de disponer de cierta variedad a la hora de escoger los recursos digitales a utilizar como componentes en las lecciones de los cursos, los proyectos MOOC utilizaron mayoritariamente las páginas html (38\% la etiqueta [html]), los vídeos ( $32 \%$ la etiqueta [video]) y, en menor medida, los problemas o ejercicios ( $15 \%$ la etiqueta [problem]) y los foros de discusión (4\% la etiqueta [discussion]) como los componentes de las unidades didácticas de cada lección del curso. Los recursos especiales de la plataforma edX tales como la evaluación por pares (la etiqueta [openassessment]), las encuestas de preguntas (la etiqueta [poll_question]) y la nube de palabras (la etiqueta [word_cloud]) no llegaron a ocupar el 1\% del volumen de componentes del curso. 


\section{Figura 2}

Frecuencia de los componentes utilizados en cada proyecto MOOC por tipo de recurso digital de la plataforma edX

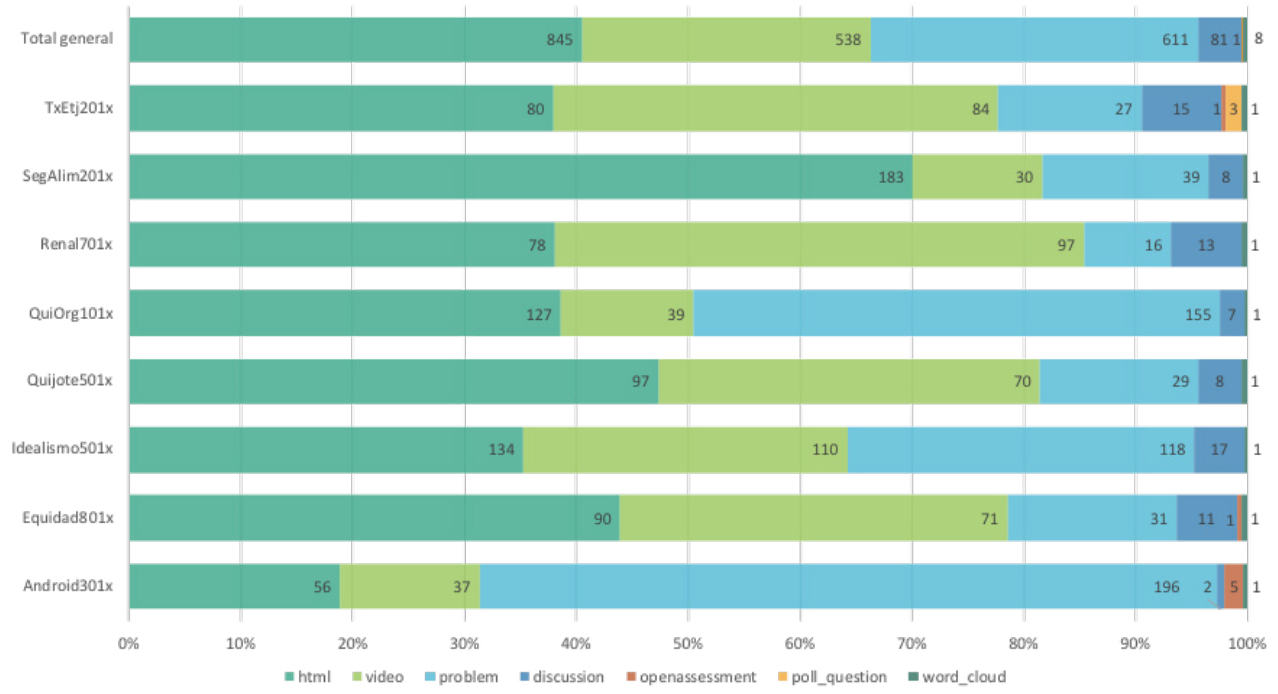

Cabe destacar, que las páginas html son el recurso más flexible entre todos los disponibles en la plataforma edX, pues permiten insertar a través de la etiqueta $[<$ iFrame $>$ ] una variedad de recursos externos dentro de las unidades didácticas en la plataforma e-learning, tales como enlaces a los blogs, las redes sociales, los documentos, otras páginas web, recursos multimedia, infografías interactivas, etc. y, por ello, con una alta incidencia en estos cursos junto con los vídeos.

Asimismo, Renal701x (47\%) y TxEtj201x (40\%) se destacan como los cursos más centrados en los componentes de las píldoras de vídeo (la etiqueta [video]), mientras Android301x (66\%) y QuiOrg101x (47\%) como los cursos más centrados en los componentes de los problemas o ejercicios de tipo test (la etiqueta [problem]).

Se observó que las declaraciones del profesorado concuerdan con los análisis estadísticos sobre la incidencia de los recursos digitales en los proyectos MOOC.

Los recursos digitales que sí utilizamos fueron evidentemente los vídeos, los cuestionarios, las imágenes con las lupas que nos gustaron mucho, creo que fueron muy interesantes [...]. (Profesor $8 \mathrm{de} \mathrm{AH}$ )

Por lo que yo recuerdo, teníamos los vídeos, la preguntas y en mí caso sí que había un ejercicio interactivo, al tener un diagrama de flujo y ser capaz de ubicar los elementos ahí, y foros donde sí estuve trasteando pues un par de veces [...]. (Profesor 16 de $\mathrm{CN}$ ) 
F. La enseñanza más atractiva con enfoque práctico a través de píldoras de contenido audiovisual también supuso un aprendizaje y un gran esfuerzo al profesorado.

De esa manera, la creación de contenido audiovisual se convirtió en el recurso más novedoso en relación con la enseñanza tradicional de estos profesores, desde la necesidad de repensar la presentación de los contenidos habituales a un formato digital, interactivo y dinámico.

[...] todo lo que aprendimos para trasmitir un mensaje a través de un vídeo, el tiempo que requiere y recomendado para transmitir el mensaje, porque si no después se pierde la atención y se pierde el mensaje, el hecho de tener que repensar mucho, pero mucho el contenido, cómo lo transmito para poderlo hacer en seis minutos, supone todo un proceso de reflexión muy profunda sobre cuáles son los contenidos imprescindibles, cuáles son los elementos centrales, qué es necesario para lo siguiente y eso es un proceso complicado que fue imprescindible introducirlo en el propio diseño del MOOC. (Profesora 14 de CS)

\section{Aspectos de la plataforma y los servicios de apoyo organizacional}

G. Pese a la ampliación de la infraestructura y de la organización laboral para la máxima automatización de los servicios de la producción audiovisual, se acusa la necesidad de más apoyo institucional y formación pedagógica.

Una de las necesidades de la enseñanza en línea es disponer de una infraestructura de apoyo a la creación de recursos docentes audiovisuales y multimedia que constituyen el principal medio de la docencia virtual. Por ello, la UTED realizó un gran esfuerzo para incorporar el espacio y la infraestructura tecnológica necesaria a este propósito. En junio de 2016, pone a disposición del profesorado, las tecnologías para crear recursos audiovisuales y multimedia que apoyen a su docencia y para facilitar el desarrollo de tecnologías educativas en el Campus. El espacio de creación de recursos docentes audiovisuales y multimedia para el apoyo a la docencia de la UAM cuenta principalmente con una Sala Polimedia. Ese sistema fue diseñado y exportado por la Universitat Politècnica de València (UPV) a la UAM para la creación de contenido educativo multimedia de alta resolución de forma económica y sencilla que permite integrar en la misma grabación, sin necesidad de edición posterior, la explicación realizada por el profesor junto con los materiales de apoyo visual que precise para ilustrar su exposición.

En ese sentido, los profesores reconocieron los esfuerzos de los responsables de la administración universitaria en promover la ampliación de la infraestructura y de la organización laboral de la oficina UAMx de cara a ofrecer la máxima automatización 
de los servicios de apoyo a la producción audiovisual al profesorado de los MOOC, SPOC y otros proyectos de innovación, aunque destacaron debilidades en los servicios de apoyo del personal técnico y especializado en audiovisuales.

insuficiente! No hubo personal calificado para realizar toda la grabación audiovisual y de la captura de pantalla con las instrucciones de programación. Se realizó por el propio personal docente con la herramienta Camtasia, sin conocimientos adecuados de definición de imagen y demás, aunque al final hubo alguna ayuda en el montaje de las imágenes de los vídeos con los audios. (Profesor 17 de IT)

Por otra parte, se criticó la falta de una formación pedagógica en lugar del enfoque tan instrumental de la formación caracterizada por los aspectos estéticos de la producción de los contenidos digitales, así como la necesidad de tiempo para asimilación y autonomía en estas nuevas competencias.

[...] en términos de diseño gráfico y de despliegue audiovisual para que ellos hicieran la edición, una parte era que no sabíamos, otra parte es que los cursos [recibidos] no nos hicieron tan competentes como para poder hacerlos bien y lo que si tomamos conciencia era que hacerlo bien llevaba tiempo, entonces aún estamos en ese tránsito. (Profesor 19 de CS).

H. La navegación y organización de los contenidos y actividades, por secuencias de aprendizaje, fue un aspecto difícil de asimilar por parte el profesorado.

En primer lugar, cabe indicar que el profesorado implicado ha utilizado como referencia en sus reflexiones sobre el diseño, la funcionalidad y la eficacia de la plataforma MOOC sus propios antecedentes y experiencias previas en otras plataformas educativas, especialmente Moodle, la plataforma institucional oficial de la UAM.

Su valoración general de la plataforma edX fue positiva, aunque reconocieron algunas limitaciones en sus funcionalidades y las propias limitaciones en el conocimiento y manejo de esta. Asimismo, algunos profesores valoraron la navegación como poco intuitiva y atractiva al estudiante, principalmente debido al concepto de secuencia de aprendizaje que hace referencia a la forma del desplazamiento horizontal por las unidades didácticas del contenido del curso.

Está bien, pero es poco intuitiva [...] porque cuando yo hice un curso de Coursera, y luego gente que conozco que ha hecho el MOOC nuestro, que han hecho algún curso Coursera, que es la más conocida, la gente dice que es más fácil guiarte en Coursera, $[\ldots . .$.$] gente que dejó partes sin hacer que luego tuvo que volver porque$ 
no lo había visto, entonces creo la gente está acostumbrada a ir hacia abajo y no hacia la derecha, entonces pensaban que habían acabado, pero no habían hecho nada, y me han dicho que la plataforma les ha resultado menos intuitiva que la otra. (Profesora 5 de CSd)

\section{CONCLUSIONES}

Los resultados del estudio de caso coinciden con investigaciones anteriores (Czerniewicz et al., 2016; Mallmann y Nobre, 2017; Misra, 2018) sobre el impacto positivo en la mejora del desarrollo profesional docente a partir de los conocimientos prácticos adquiridos en la experiencia de innovación tecnológica a través de los MOOC.

Desde la percepción del profesorado implicado en estos proyectos MOOC, se reconoció que la experiencia de innovación tecnológica aportó cambios y mejoras en su formación pedagógica, principalmente con relación a la capacidad de comunicarse eficientemente en un formato audiovisual. Ayudó a reflexionar sobre un nuevo papel docente digital como mediador y dinamizador del aprendizaje. Asimismo, el profesorado valoró más el aprendizaje de las habilidades de la educación mediática y la planificación de las actividades digitales que el manejo instrumental de los recursos y plataformas tecnológicas.

Adicionalmente, se observó que el creciente interés de los profesores por los entornos digitales viene acompañado por un completo desconocimiento sobre los aspectos pedagógicos de las prácticas abiertas de la enseñanza en línea y las posibilidades de utilizar los contenidos multimedia interactivos para fomentar nuevos modelos de interacción didáctica. Tales contradicciones hicieron muy desafiante para el profesorado llevar a cabo estas experiencias de innovación docente con tecnologías educativas.

Los análisis evidenciaron otros tres puntos críticos en este proceso: la formación pedagógica en competencias de la educación mediática, la brecha digital y la sobrecarga de trabajo. Por lo tanto, resulta fundamental más inversión institucional y mejor reconocimiento de la actividad de innovación docente con tecnologías digitales para reforzar sus oportunidades y solventar las dificultades que implica a la reconstrucción de la identidad docente y a la renovación de los modelos de enseñanza superior (Area, 2018; Ferrés et al., 2018; García Peñalvo y Corell, 2020; Sancho et al., 2018). Para ello, los servicios institucionales deberán no solo garantizar las estructuras tecnológicas, sino mejorar la formación pedagógica y apoyo técnico de equipos multidisciplinares de cara a trabajar la alfabetización audiovisual y mediática del profesorado.

Para seguir profundizando en torno a los cambios pedagógicos a través de los entornos digitales, se recomienda continuar con estudios interpretativos sobre los procesos de la renovación de los programas de innovación docente con tecnologías educativas, que incluye las políticas de calidad de la enseñanza, el reconocimiento 
de carga laboral en innovación docente, así como el liderazgo académico para una cultura de cambio desde la implicación y reflexión del profesorado agente del cambio.

\section{REFERENCIAS BIBLIOGRÁFICAS}

Adell, J., Castañeda, L., y Esteve, F. (2018). ¿Hacia la Ubersidad? Conflictos y contradicciones de la universidad digital. RIED. Revista Iberoamericana de Educación a Distancia, 21(2), 51-68. https://doi.org/10.5944/ried.21.2.20669

Anderson, T., y Dron, J. (2012). Learning technology through three generations of technology enhanced distance education pedagogy. European Journal of Open, Distance and E-Learning, 1-14. http:// hdl.handle.net/2149/3204

Anderson, T., y Mcgreal, R. (2012). Disruptive pedagogies and technologies in universities. Educational Technology and Society, 15(4), 380-389.

Angrosino, M. (2012). Etnografía y observación participante en Investigación Cualitativa (M. del C. Blanco Castellano y T. Amo Martín (Eds.), (1. ${ }^{\mathrm{a}}$ ed.). Ediciones Morata.

Area, M. (2018). De la enseñanza presencial a la docencia digital. Autobiografía de una historia de vida docente. Revista de Educación a Distancia (RED), 56(1), 1-21. https://doi.org/10.6018/red/56/1

Armellini, A., y Padilla-Rodriguez, B. C. (2016). Are Massive Open Online Courses (MOOCs) pedagogically innovative? Journal of Interactive Online Learning (JIOL), 14(1), 17-28.

Bai, Y., Li, H., y Liu, Y. (2021). Visualizing research trends and research theme evolution in E-learning field: 19992018. Scientometrics, 126(2), 1389-1414. https://doi.org/10.1007/s11192-02003760-7

Bartolomé, A.-R., y Steffens, K. (2015). Are MOOCs Promising Learning Environments? Comunicar - Revista Científica de Educomunicación, 22(44),
91-99. $\quad$ https://doi.org/10.3916/C442015-10

Cabero, J., y Llorente, M. del C. (2017). Los MOOC: encontrando su camino.@ tic. revista d'innovació educativa, EneJun(18), 24-30. https://doi.org/10.7203/ attic.18.9928

Callejo-Gallego, J., y Agudo-Arroyo, Y. (2018). MOOC: valoración de un futuro. RIED. Revista Iberoamericana de Educación a Distancia, 21(2), 219-241. https://doi.org/10.5944/ried.21.2.20930

Chiappe-Laverde, A., Hine, N., y MartínezSilva, J. A. (2015). Literature and Practice: A Critical Review of MOOCs. Comunicar Revista Científica de Educomunicación, 22(44), 09-18. https://doi.org/10.3916/ C44-2015-01

Chinkes, E., y Julien, D. (2019). Las instituciones de educación superior y su rol en la era digital. La transformación digital de la universidad: ¿transformadas o transformadoras? Ciencia y Educación, 3(1), 21-33. https://doi.org/10.22206/ cyed.2019.v3i1.pp21-33

Conole, G. (2016). MOOCs as disruptive technologies: strategies for enhancing the learner experience and quality of MOOCs. Revista de Educación a Distancia (RED), 39(50), 1-17. https://doi.org/10.6018/ $\mathrm{red} / 5 \mathrm{O} / 2$

Corny, D. (2021, abril 9). Position of the Educational Technologist in Creating Videos for a MOOC. MOOCs, Language learning and mobility, design, integration, reuse, Apr 2021, Online Conference. $\quad$ https://hal.archivesouvertes.fr/hal-03232153

Creswel, J. W., y Clark, V. L. P. (2017). Designing and Conducting Mixed 
Methods Research (3. ${ }^{\mathrm{a}}$ ed.). SAGE Publications.

Czerniewicz, L., Glover, M., Decano, A., y Sukaina, W. (2016). MOOCs, openness and changing educator practices: an Activity Theory case study. En S. Cranmer, N. B. Dohn, M. de Laat, T. Ryberg, y J. A. Sime (Eds.), Proceedings of the 1oth International Conference on Networked Learning 2016 (pp. 287-294). Department of Educational Research at Lancaster University. http://www. networkedlearningconference.org.uk/ abstracts/pdf/P26.pdf

Daniel, J., y Uvalić-Trumbić, S. (2014). Are MOOCs the long-awaited technological revolution in higher education? Digital Transformations Conference. https:// oerknowledgecloud.org/content/aremoocs-long-awaited-technologicalrevolution-higher-education

Demetriadis, S., Karakostas, A., Tsiatsos, T., Caballé, S., Dimitriadis, Y., Weinberger, A., Papadopoulos, P. M., Palaigeorgiou, G., Tsimpanis, C., y Hodges, M. (2018). Towards Integrating Conversational Agents and Learning Analytics in MOOCs. En L. Barolli, F. Xhafa, N. Javaid, E. Spaho, y V. Kolici (Eds.), Advances in Internet, Data \& Web Technologies. EIDWT 2018. Lecture Notes on Data Engineering and Communications Technologies (pp. 1061-1072). Springer, Cham. https://doi. org/10.1007/978-3-319-75928-9 98

Downes, S. (2012). E-Learning Generations. Stephen's Web. https://www.downes.ca/ post/57741

Drake, J. R., O'Hara, M. T., y Seeman, E. (2015). Five Principles for MOOC Design: With a Case Study. Journal of Information Technology Education: Innovations in Practice, 14, 125-143. https://doi.org/10.28945/2250

Ebben, M., y Murphy, J. S. (2014). Unpacking MOOC scholarly discourse: a review of nascent MOOC scholarship. Learning, Media and Technology, 39(3), 328-345. https://doi.org/10.1080/17439884.2013. $\underline{878352}$

Elbaz, F. (1983). Teacher Thinking: a study of practical knowledge. Croom Helm.

Ferrés, J., Masanet, M.-J., y Mateus, J.-C. (2018). Three paradoxes in the approach to educational technology in the education studies of the Spanish universities. International Journal of Educational Technology in Higher Education, 15(1), 15. https://doi.org/10.1186/s41239-0180097-y

Flick, U. (2002). An introduction to Qualitative Research (2. ${ }^{\mathrm{a}}$ ed.). SAGE Publications.

García Peñalvo, F. J., y Corell, A. (2020). La CoVId-19: ¿enzima de la transformación digital de la docencia o reflejo de una crisis metodológica y competencial en la educación superior? Campus Virtuales, 9(2), 83-98. http://hdl.handle. net/10366/144140

Gehrke, S., y Kezar, A. (2015). Unbundling the Faculty Role in Higher Education: Utilizing Historical, Theoretical, and Empirical Frameworks to Inform Future Research. En M. B. Paulsen (Ed.), Higher Education: Handbook of Theory and Research (93-150). Springer. https://doi. org/10.1007/978-3-319-12835-1

Gértrudix Barrio, M., Rajas Fernández, M., y Álvarez García, S. (2017). Metodología de producción para el desarrollo de contenidos audiovisuales y multimedia para MOOC. RIED. Revista Iberoamericana de Educación a Distancia, 2o(1), 183. https://doi. org/10.5944/ried.20.1.16691

Giasiranis, S., y Sofos, L. (2020). The Influence of Instructional Design and Instructional Material on Learners' Motivation and Completion Rates of a MOOC Course. Open Journal of Social Sciences, o8(11), 190-206. https://doi. org/10.4236/jss.2020.811018

Gil-Jaurena, I., y Domínguez, D. (2018). Teachers' roles in light of massive open 
online courses (MOOCs): Evolution and challenges in higher distance education. International Review of Education, 64(2), 197-219. https://doi.org/10.1007/ s11159-018-9715-0

Guàrdia, L., Maina, M., y Sangrà, A. (2013). MOOC Design Principles. A Pedagogical Approach from the Learner's Perspective. eLearning Papers, 33(May), 1-6. http:// hdl.handle.net/10609/41681

Guo, P. J., Kim, J., y Rubin, R. (2014). How video production affects student engagement. Proceedings of the first ACM conference on Learning @ scale conference - L@S '14, 41-50. https://doi. org/10.1145/2556325.2566239

Hernández-Sampieri, R., y Mendoza, C. P. (2018). Metodología de la investigación. Las rutas cuantitativa, cualitativa $y$ mixta (1. ${ }^{a}$ ed.). Editorial McGraw Hill Education.

Hollands, F., y Tirthali, D. (2014). MOOCs: Expectations and Reality. En Full report (May). https://files.eric.ed.gov/fulltext/ ED547237.pdf

Kuzu, Ö. H. (2020). Digital Transformation in Higher Education: A Case Study on Strategic Plans. Vysshee Obrazovanie $v$ Rossii $=$ Higher Education in Russia, 29(3), 9-23. https://doi. org/10.31992/0869-3617-2019-29-3-9$\underline{23}$

Kvale, S. (2011). Las entrevistas en investigación cualitativa. En M. del C. Blanco Castellano y T. Amo Martín (Eds.); (1. ${ }^{\mathrm{a}}$ ed.). Ediciones Morata.

Lane, L. M. (2012). Three Kinds of MOOCs. Lisa's (Online) Teaching \& History Blog. http://lisahistory.net/ wordpress/2012/08/three-kinds-ofmoocs/

Lemay, D. J., y Doleck, T. (2020). Predicting completion of massive open online course (MOOC) assignments from video viewing behavior. Interactive Learning Environments, 1-12. https://doi.org/10.1 080/10494820.2020.1746673
Lin, J. (2017). Exploring the experiences of instructors teaching massive open online courses in tourism and hospitality [Università della Svizzera italiana]. http://doc.rero.ch/record/306564

Mallmann, E. M., y Nobre, A. M. (2017). Un canal abierto no encino superior? MOOC e REA no mundo digital. Apertura, 9(2), 24-41. https://doi.org/10.32870/ Ap.vgn2.1126

Misra, P. K. (2018). MOOCs for Teacher Professional Development: Reflections and Suggested Actions. Open Praxis, 10(1), 67. https://doi.org/10.5944/ openpraxis.10.1.780

Moe, R. (2015). The brief \& expansive history (and future) of the MOOC: Why two divergent models share the same name. Current Issues in Emerging eLearning (CIEE), 2(1|2), 1-14.

Nascimbeni, F., y Burgos, D. (2016). In Search for the Open Educator: Proposal of a Definition and a Framework to Increase Openness Adoption Among University Educators. The International Review of Research in Open and Distributed Learning (IRRODL), 17(6), 1-17. https:// doi.org/10.19173/irrodl.v17i6.2736

Ramírez-Fernández, M. B., SalmeronSilvera, J. L., y López-Meneses, E. (2015). Comparative between quality assessment tools for MOOCs: ADECUR vs Standard UNE 66181: 2012. Universities and Knowledge Society Journal (RUSC), 12(1), 1-15. https://doi.org/10.7238/rusc. v12i1.2258

Ramírez-Montoya, M.-S. (2020). Transformación digital e innovación educativa en Latinoamérica en el marco del COVID-19. Campus Virtuales, 9(2). http://uajournals.com/ojs/index.php/ campusvirtuales/article/view/744

Ramírez-Montoya, M.-S., y Lugo-Ocando, J. (2020). Systematic review of mixed methods in the framework of educational innovation. Comunicar, 28(65), 9-20. https://doi.org/10.3916/C65-2020-01 
Salinas, J., Pérez, A., y Benito, B. de. (2008). Metodologías centradas en el alumno para el aprendizaje en red (1. ${ }^{a}$ ed.). Síntesis.

Sánchez-Vera, M. del M., León-Urrutia, M., y Davis, H. (2015). Challenges in the Creation, Development and Implementation of MOOCs: Web Science Course at the University of Southampton. Comunicar - Revista Científica de Educomunicación, 22(44), 37-44.https:// doi.org/10.3916/C44-2015-04

Sancho, J. M. (2018). Innovación y enseñanza. De la "moda" de innovar a la transformación de la práctica docente. Educação, 41(1), 12. $\quad$ https://doi. org/10.15448/1981-2582.2018.1.29523

Sancho, J. M., Ornellas, A., y Arrazola, J. (2018). La situación cambiante de la universidad en la era digital. RIED. Revista Iberoamericana de Educación a Distancia, 21(2), 31-49. https://doi. org/10.5944/ried.21.2.20673

Sangrà, A., González-Sanmamed, M., y Anderson, T. (2015). Metaanálisis de la investigación sobre mooc en el período 2013-2014. Educacion XX1, 18(2), 21-49. https://doi.org/10.5944/educxx1.13463

Selwyn, N. (2018). What is Digital Sociology? (1. ${ }^{\mathrm{a}}$ ed.). Polity Press.

Siemens, G. (2013). Massive Open Online Courses: Innovation in Education? En R. McGreal, W. Kinuthia, y S. Marshall (Eds.), Open Educational Resources: Innovation, Research and Practice (1. ${ }^{\mathrm{a}}$ ed.), (5-15). Commonwealth of Learning and Athabasca University.

Silva, V., y Souza, R. (2016). E-learning, B-learning, M-learning and the technical and pedagogical aspects on the new platform trends as massive open online courses. ICERI2O16 Proceedings, (pp. 5521-5529). https://doi.org/10.21125/ iceri.2016.0239
Stake, R. E. (2010). Qualitative Research: Studying How Things Work (1 $\left.{ }^{\mathrm{a}}\right)$. Guilford Press. https://doi.org/10.1017/ CBO9781107415324.004

Strauss, A., y Corbin, J. (2002). Bases de la investigación cualitativa. Técnicas y procedimientos para desarrollar la teoría fundamentada. En E. Zimmerman (Ed.) (1. ${ }^{a}$ ed.). Editorial Universidad de Antioquia.

Tardif, M. (2004). Los saberes del docente y su desarrollo profesional. Narcea.

Toven-Lindsey, B., Rhoads, R. A., y Lozano, J. B. (2015). Virtually unlimited classrooms: Pedagogical practices in massive open online courses. The Internet and Higher Education, 24, 1-12. https:// doi.org/10.1016/j.iheduc.2014.07.001

Vázquez-Cano, E., López-Meneses, E., Gómez-Galán, J., y Parra-González, M. E. (2021). Prácticas universitarias innovadoras sobre las ventajas educativas y desventajas de los entornos MOOC. Revista de Educación a Distancia (RED), 21(66), 30-2021. https://doi. org $/ 10.6018 /$ red.422141

Zapata-Ros, M. (2013). MOOCs, una visión crítica y una alternativa complementaria: La individualización del aprendizaje y de la ayuda pedagógica. Campus Virtuales | Revista Cientifica de Tecnología Educativa, 2(1), 20-38.

Zhao, Y., Wang, A., y Sun, Y. (2020). Technological environment, virtual experience, and MOOC continuance: A stimulus-organism-response perspective. Computers \& Education, 144, 103721. https://doi.org/10.1016/j. compedu.2019.103721

Zhu, M., Bonk, C., y Sari, A. (2018). Instructors' Experience of Designing MOOCs in Higher Education: Considerations and Challenges. Online Learning, 22(4), 203-241. https://doi. org/10.24059/olj.v22i4.1495 


\section{PERFIL ACADÉMICO Y PROFESIONAL DE LOS AUTORES}

Ada Freitas Cortina es Doctora en Educación y Máster en Tecnologías de la Información y la Comunicación en Educación y Formación. Profesora asociada al Departamento de Pedagogía de la Universidad Autónoma de Madrid (UAM, España) donde imparte docencia en grado y posgrado en el área de Tecnologías para la Educación. Miembro del grupo de investigación EMIPE (Equipo de Mejora Interdisciplinar para las Prácticas Educativa) de la UAM. Sus líneas de investigación giran en torno a la educación mediática, competencia digital, e-learning, tecnologías educativas e innovación docente en la enseñanza superior y la formación del profesorado. https://orcid.org/0000-0002-9731-4641

E-mail: ada.freitas@uam.es

Joaquín Paredes Labra es Doctor en Pedagogía. Profesor Titular en el Departamento de Pedagogía de la Facultad de Formación de Profesorado y Educación de la Universidad Autónoma de Madrid (UAM, España). Enseña sobre tecnología educativa y ha colaborado con numerosas universidades españolas en el desarrollo de cursos sobre esta temática. También ha colaborado con diversas universidades y entidades por la ciencia latinoamericanas, con cursos sobre el papel del docente contemporáneo, narrativas digitales, blended learning y los procesos de utilización de las TIC en Educación en Valores y Educación Ambiental. Miembro de varios comités científicos, entre otros el Asesor de Formación Virtual de la UAM (2008-10), Directivo de la sociedad científica RUTE (2006-12). Miembro de varias sociedades científicas. Sus líneas de investigación en el grupo de investigación EMIPE de la UAM versan sobre formación pedagógica inicial y continua del profesorado, tecnología educativa, didáctica e innovación. Autor de más de un centenar de trabajos, entre sus libros está el más reciente: Didáctica inclusiva y transformadora (2019, Síntesis). https://orcid.org/0000-0003-2294-9121

E-mail: joaquin.paredes@uam.es

Fecha de recepción del artículo: 08/04/2021

Fecha de aceptación del artículo: 06/07/2021

Fecha de aprobación para maquetación: 06/09/2021 